\title{
Elephantiasis Nostras Verrucosa in leprosy.
}

\author{
Sonam Goyal ${ }^{1}$, S.N Mahajan ${ }^{2}$, Sourya Acharya ${ }^{3}$, Adarsh Lata Singh ${ }^{4}$ \\ 1. Resident, Dept of Medicine \\ 2. Professor and HOD, Dept of Medicine \\ 3. Professor, Dept of Medicine \\ 4. Professor and HOD, Dept of Dermatology and Venereal Diseases.
}

\begin{abstract}
We present a case of Elephantiasis Nostras Verrucosa in a patient of leprosy with peripheral neuropathy.

Key Words: ENV, Leprosy, peripheral neuropathy

\section{Introduction:}

Elephantiasis nostras verrucosa (ENV) is the progressive disfiguring enlargement of a body part caused by recurrent soft tissue bacterial infections in the setting of chronic secondary lymphedema. The basic predisposing factor its development is lymphatic obstruction in any part of the body. This obstruction may be primary or secondary due to long standing non lymphatic edematous conditions that ultimately lead to secondary obstruction to lymph flow. Secondary infections due to poor vascularity, neuropathy and lymphedema further aggravates the clinical situation.
\end{abstract}

\section{Case Report:}

A 70 Years old male patient presented to us with chief complain of swelling and disfigurement, foul smelling discharge in bilateral lower limbs since 1 month. He was diagnosed case of lepromatous leprosy and was on anti leprosy treatment since 8 months. At the time of the diagnosis of leprosy his nerve conduction study had been done which revealed demyelinating type of sensory neuropathy.

On examination there was bilateral woody swelling in the foot extending up to mid calves with a circumferential confluence of white- to pink-coloured plaques with verrucous, "cobblestoned" appearing tightly packed, tense papules with ulcerations and foul smelling foul smelling discharge were seen on dorsum of foot typical of ENV.[figure 1]

On investigation; Complete blood count was normal, Heterazan challenge test was negative, Doppler lower limb suggested decreased flow velocity in the superficial lower limb veins suggesting venous stasis. Culture growth of discharge showed staphylococci organisms. Patient was started on Tab Augmentin $625 \mathrm{mg}$ tds for 7 days and topical retinoids was given. Along with it compression stocking to calves was applied. After which discharge reduced. Provisional diagnosis of Elephantiasis Nostras Verrucosa (ENV) was made.

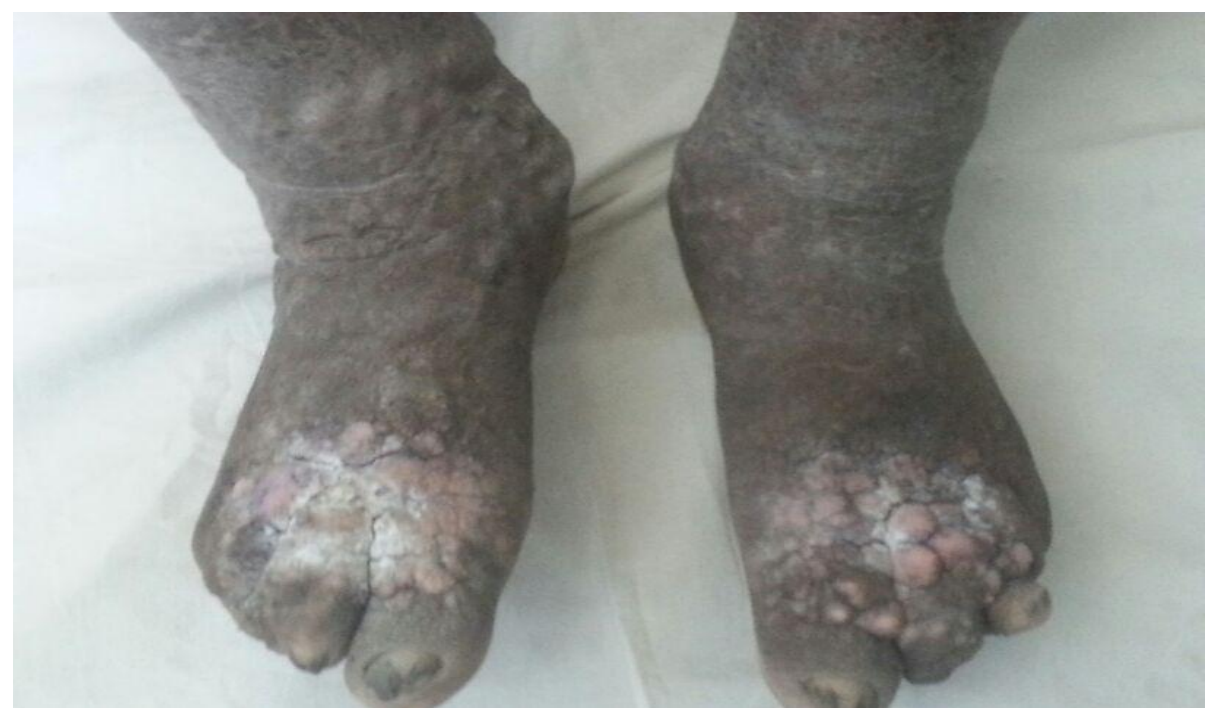

Figure 1; Showing typical lesion of Elephantiasis Nostras Verrucosa. Also seen are the destruction of the digits secondary to long standing leprosy. 


\section{Discussion:}

ENV is a form of chronic secondary lymphedema; primary lymphedema is caused by congenital defects in the lymphatic system ${ }^{[1]}$.Secondary lymphedema is the dysfunction of the lymphatic system caused by another primary disease process. Primary infectious etiologies include filarial, staphylococcal, and streptococcal infections, which cause direct inflammation and fibrosis of the lymphatic vessels. Noninfectious etiologies include disruption of the lymphatic system after trauma, surgery, obstruction by malignancy, CHF, and obesity, and lymphatic fibrosis by malignancy, radiation, venous stasis, CHF, obesity, portal hypertension, and scleroderma.

It is currently unclear which patients with chronic lymphedema progress to ENV. It is hypothesized that the protein-rich fluid that accumulates in the interstitium provokes an inflammatory response that impairs the local immune response and predisposes the patient to soft tissue infections such as erysipelas, cellulitis, and lymphangitis. Inoculation can result from insignificant trauma, poor hygiene, and dry fissured skin ${ }^{[2]}$ With each bout of soft-tissue infection the lymphatics become increasingly fibrotic and the affected body part becomes more edematous and enlarged, even after the acute infection has subsided. Meanwhile, the skin undergoes epidermal hyperkeratosis and fibrosis of the dermis and subcutaneous tissues. ${ }^{[3]}$ In time this produces the characteristic clinical appearance of ENV. Our patient was a case of lepromatous leprosy with peripheral neuropathy, secondary venous stasis, neuropathic recurrent traumatic ulcers, and with lymphedema which may be secondary to leprosy or its treatment with clofazimine which is known to cause lymphadema. All these factor may have contributed to ENV.

\section{Management}

There currently is no established standard of care for the treatment of ENV. Therapies attempt to correct the underlying cause, maximize the patient's ability to use the affected limb, and prevent further complications. ${ }^{[4]}$ Initial conservative treatments include elevation of the affected limb, compression with bandages or stockings, massage, and pneumatic compression devices ${ }^{[5]}$ During acute lymphangitis, appropriate antibiotics should be used to treat the infection. When there is no active infection, prophylactic use of antibiotics may be considered. There are several reports of success with the use of oral and topical retinoids, such as etretinate and tazarotene, respectively, which decrease epidermal proliferation, fibrogenesis, and inflammation. If medical measures fail, surgical options are available. These include debridement, lymphatic and lymphovenous anastomosis, lymphatic transplantation and, for severe cases in which the outlook of limb preservation is bleak, amputation.

\section{Conclusion}

Our patient had no identifiable malignancies, history of radiation therapy, surgical history, history of travel to tropical regions, or family history of similar pathology that would have caused his lymphedema. His leprosy, however, contributed significantly to his lymphedema and resulting ENV.

\section{References}

[1]. Tiwari A, Cheng K, Button M, Myint F, Hamilton G. Differential diagnosis, investigation, and current treatment of lower limb lymphedema. Arch Surg 2003; 138: 152.

[2]. Schissel DJ, Hivnor C, Elston DM. Elephantiasis nostras verrucosa. Cutis 1998; 62: 77-80.

[3]. Sisto K, Khachemoune A. Elephantiasis nostras verrucosa: a review. Am J Clin Dermatol 2008; 9: 141-6.

[4]. Turhan E, Ege A, Keser S, Bayar A. Elephantiasis nostras verrucosa complicated with chronic tibial osteomyelitis. Arch Orthop Trauma Surg 2007; 128: 1183-6.

[5]. Iwao F, Sato-Matsumura KC, Sawamura D, Shimizu H. Elephantiasis nostras verrucosa successfully treated by surgical debridement. Dermatol Surg 2004; 30: 939-41. 